\title{
Réforme relative à l'équilibre entre le travail flexible et le travail permanent
}

\section{Nicola Gundt}

\section{Q OpenEdition}

1 Journals

Édition électronique

URL : https://journals.openedition.org/rdctss/1692

DOI : 10.4000/rdctss. 1692

ISSN : 2262-9815

Éditeur

Centre de droit comparé du travail et de la sécurité sociale

\section{Édition imprimée}

Date de publication : 1 avril 2019

Pagination : 226-229

ISSN : 2117-4350

\section{Référence électronique}

Nicola Gundt, « Réforme relative à l'équilibre entre le travail flexible et le travail permanent », Revue de droit comparé du travail et de la sécurité sociale [En ligne], 1 | 2019, mis en ligne le 01 novembre 2021, consulté le 13 novembre 2021. URL : http://journals.openedition.org/rdctss/1692 ; DOI : https:// doi.org/10.4000/rdctss. 1692

\section{(c) (†) $९$}

Revue de droit comparé du travail et de la sécurité sociale est mise à disposition selon les termes de la Licence Creative Commons Attribution - Pas d'Utilisation Commerciale - Pas de Modification 4.0 International. 


\section{RÉFORME RELATIVE À L'ÉQUILIBRE ENTRE LE TRAVAIL FLEXIBLE ET LE TRAVAIL PERMANENT}

Après avoir remporté les élections, le gouvernement s'est engagé à remanier le droit du travail néerlandais qui avait été réformé en 2015¹. Il s'est efforcé de trouver un nouvel équilibre entre emploi flexible et emploi permanent. Pour ce faire, il a décidé de limiter la durée maximale du contrat à durée déterminée renouvellement compris. L'autre mesure principale consistait à accélérer la procédure de licenciement, à la faciliter et à le rendre, moins coûteux, ce qui inciterait les employeurs à proposer plus facilement des contrats à durée indéterminée. Cela permettrait également d'atteindre l'autre objectif: rendre le travail flexible moins flexible, car les employeurs proposeraient plus facilement des postes permanents ${ }^{2}$.

Cependant, des recherches ont montré qu'il est devenu plus difficile de licencier des salariés - même si les coûts étaient nettement moins élevés qu'auparavant. Cela a conduit le nouveau gouvernement de coalition à présenter une proposition intitulée «Equilibrer le marché de l'emploi » (Wet Arbeidsmarkt in balans, WAB) ${ }^{3}$. L'idée centrale est de réduire les différences de coûts entre travail flexible et travail permanent / typique, afin de garantir que les types de contrat atypiques et / ou flexibles soient choisis pour des raisons de souplesse, et non en raison du coût ${ }^{4}$. Baser la concurrence sur le type de contrat utilisé doit être autant que possible écarté. Par conséquent, les employeurs paieront des primes selon le type de contrat en fonction du risque de chômage: plus élevée pour un travail flexible, moins élevée pour un travail à durée indéterminée. En outre, l'indemnité de licenciement prévue par la loi (transitievergoeding) sera due à compter du premier jour d'emploi, ce qui égalisera les coûts des différents types de contrats. Outre ces idées concernant les coûts de l'emploi, la proposition contient deux séries de mesures: I'une portant sur le travail flexible (A), l'autre sur le travail à durée indéterminée (B).

\section{A - LeS CHANGEMENTS CONCERNANT LE TRAVAIL FLEXIBLE}

Un premier changement majeur est le fait qu'à partir du $1^{\text {er }}$ janvier 2020, l'employeur doit payer l'indemnité de départ prévue par la loi à compter du premier jour d'emploi. Jusque-là, en règle générale, l'indemnisation n'était due que si le salarié avait travaillé pendant au moins 24 mois pour un même employeur. Etant donné que la durée maximale d'autorisation des contrats à durée déterminée, renouvellement compris est actuellement de 24 mois, il n'est pas surprenant que dans la majorité des cas, les contrats à durée

1 Regeerakkoord "Vertrouwen in de toekomst".

https://www.tweedekamer.nl/debat_en_vergadering/uitgelicht/kabinetsformatie-2017.

2 Wet werk en Zekerheid, TK 2013/14, 33 818, n 3.

3 TK 2018/19, $35074, n^{\circ} 3$

4 Ibid., p.8. 
déterminée ne soient pas transformés en contrats à durée indéterminée à cause des coûts. Le gouvernement espère que cette égalisation des coûts incitera les employeurs à faire un choix qui ne repose pas uniquement sur le coût et qu'ils tiendront compte d'autres aspects, tels que l'importance du salarié pour l'entreprise: dans quelle mesure est-il remplaçable?

Un deuxième changement envisagé semble toutefois être diamétralement opposé : la période maximale des contrats à durée déterminée, renouvellements compris, est (à nouveau) prolongée de 24 à 36 mois. Ce changement ne s'explique pas facilement. Le marché de l'emploi néerlandais fonctionne bien, le chômage est au plus bas et la période maximale n'a été réduite qu'en 2015, afin de permettre aux personnes d'obtenir un emploi permanent plus rapidement. A l'époque, tous les spécialistes du droit du travail avaient prévenu que la combinaison d'un plafonnement maximum de 24 mois pour le contrat de travail à durée déterminée et le déclenchement du droit à l'indemnité de licenciement audelà de 24 mois d'ancienneté irait dans le sens d'un renforcement mutuel des deux mesures 5 . Il aurait donc été intéressant de voir l'impact sur le comportement des employeurs du nouveau droit à l'indemnité de licenciement dès le premier jour de travail avant d'inverser de nouveau les règles sur le renouvellement des contrats à durée déterminée. Cela est d'autant plus vrai que les arguments du gouvernement en faveur d'un allongement de la durée maximale du contrat de travail à durée déterminée ne sont pas convaincants, surtout eu égard aux excellents chiffres du marché de l'emploi.

Un troisième changement concerne les contrats sur appel, qui sont souvent moins irréguliers que leur nom ne semble l'indiquer ${ }^{6}$. Le gouvernement propose de rendre plus strictes d'assouplir les règles y relatives. Afin d'offrir plus de sécurité (économique), la proposition prévoit l'obligation de faire appel au salarié au moins quatre jours avant le jour où il est censé commencer à travailler ${ }^{7}$. Par convention collective, le délai de 4 jours peut être réduit à un jour. L'employeur doit notifier au salarié numériquement ou par écrit l'appel et les heures prévues. Cela donne au salarié la possibilité d'approuver l'appel et c'est par conséquent un motif de réclamer le salaire si l'employeur refuse plus tard de le laisser travailler ou annule l'appel. Enfin, à partir du $13^{\text {ème }}$ mois d'emploi, l'employeur doit proposer un contrat avec un minimum garanti d'heures de travail. Le droit au salaire ne peut plus être exclu s'il n'y a pas de travail. Ce nombre minimal d'heures correspond à la moyenne des heures travaillées les douze derniers mois.

Selon le gouvernement, les mesures susmentionnées créeront un nouvel équilibre en ce qui concerne les coûts de la flexibilité. Cet instrument réglementaire des coûts devrait alors inciter les employeurs à recourir à des contrats souples uniquement dans les cas où il s'agit d'une nécessité réelle et non parce que c'est simplement une solution plus commode.

5 E.g. A.R. Houweling, Wetsvoorstel werk en zekerheid en de arbeidsovereenkomst voor bepaalde tijd: over het nieuwe jaarcontract van acht maanden, TAP 2015, p. 27; A.R. Houweling, M.J.M.T. Keulaerds, P. Kruit: VAAN-VvA Evaluatieonderzoek WWZ, BjU Den Haag 2016, p. 61.

6 CBS, Flexibele Arbeid 2018.

7 TK 2018/19, 35 074, n³, p. 24. 


\section{B. LES CHANGEMENTS CONCERNANT LE TRAVAIL PERMANENT}

Ici, l'idée est de le rendre moins permanent ou, à tout le moins, de rendre le licenciement moins difficile. Selon le gouvernement cela incitera les employeurs à proposer plus rapidement des contrats à durée indéterminée - c'est l'idée qui sous-tend le concept américain de l'emploi de gré à gré.

Un premier changement concerne la période d'essai. Jusqu'ici, la durée maximale absolue de la période d'essai était de deux mois, dans le cas d'un contrat de plus de deux ans ou selon les dispositions d'une convention collective. Ceci est perçu comme trop court. Les nouvelles règles prévoient une période d'essai maximale de cinq mois pour les contrats de deux ans ou plus, de deux mois pour les contrats allant jusqu'à deux ans et de trois mois pour les contrats à durée déterminée plus longs ${ }^{8}$. Dans ces conditions, il est évident que de nouvelles questions vont se poser. Par exemple, que faire des salariées enceintes? Pendant une période d'essai de deux mois, cela peut être dissimulé. Pendant une période de cinq mois, ce sera beaucoup plus difficile. Si l'employeur décide de mettre fin au contrat - ce qui est possible sans indiquer de motif - cela ne sera probablement pas jugé discriminatoire à moins que l'employeur ne soit en fait assez stupide pour dire qu'il le fait en raison de cette grossesse. Certes, la salariée peut se prévaloir de la présomption légale de discrimination, mais si l'employeur est prudent, il y a de fortes chances pour que cette cessation d'emploi reste légale. Ce type de problèmes n'a pas été abordé dans les travaux préparatoires, bien que le gouvernement ait déjà décelé un possible abus de période d'essai plus longue. Par exemple, pour empêcher que les périodes d'essai ne soient utilisées comme une sorte de contrat à durée déterminée, la proposition ne prévoit qu'une seule période de cinq mois. En cas de changement d'emploi dans la même entreprise nécessitant des connaissances et des compétences différentes, la période d'essai maximale est de deux mois au lieu d'une période complète de cinq mois si le salarié changeait d'entreprise. De plus, un contrat à durée indéterminée, qui prend fin au cours de la période d'essai, sera traité comme un contrat à durée déterminée pour ce qui est des renouvellements de contrats consécutifs? Reste à savoir si les possibilités de résilier un contrat à durée indéterminée pendant la période d'essai (longue) conduisent réellement à conclure un plus grand nombre de contrats à durée indéterminée.

Un autre changement intéressant en ce qui concerne les contrats à durée indéterminée concerne le droit du licenciement. C'est seulement en juillet 2015 qu'une liste limitative de motifs de licenciement a été introduite. Avant de congédier un salarié, l'employeur doit prouver qu'il a un motif valable. S'il n'y parvient pas le contrat ne peut être résilié. C'est un énorme changement par rapport à l'ancien système, sous l'égide duquel la résiliation du contrat par le juge était presque toujours possible, mais pouvait coûter beaucoup si elle n'était pas justifiée ou si les raisons semblaient fragiles au juge. Afin de réduire les coûts, on a substitué à l'ancien système une indemnité légale de licenciement ainsi qu'une éventuelle indemnité supplémentaire, cette dernière étant exclusivement versée si l'employeur s'avérait gravement fautif. Dans le système actuel, deux choses vont de pair: la raison / le motif admissible et la possibilité de mettre fin au contrat si la réintégration à un autre poste dans l'entreprise s'avère impossible. Selon le gouvernement, les statistiques des trois dernières années montrent que le licenciement est devenu moins coûteux, mais

8 TK 2018/19, $35074, n^{\circ} 3$, p. 59.

9 lbidem. 
plus difficile en raison de l'incapacité des employeurs d'attester d'un motif valable. Cela est peut-être dû au fait que les employeurs étaient peu habitués à motiver et à prouver. Au lieu d'attendre, le gouvernement a décidé d'adapter le système. L'idée est qu'à partir de 2020, un employeur puisse demander la dissolution du contrat de travail par le juge s'il existe plusieurs raisons ou motifs, mais sans qu'aucun d'entre eux ne soit suffisant en soi pour justifier un licenciement ${ }^{10}$. Les motifs concernant la personne et / ou le comportement du salarié peuvent être considérés de manière cumulative. II est intéressant de noter que le gouvernement semble considérer que ce type de licenciement fondé sur des «demimotifs » cumulés ne constitue pas un licenciement pleinement justifié. Après tout, la proposition contient l'idée d'une indemnité de licenciement plus élevée, du fait que les motifs en tant que tels sont insuffisants ${ }^{11}$. A notre avis, il est étonnant que le gouvernement ouvre cette voie. Soit un licenciement est légalement possible, et alors il ne devrait y avoir aucune raison de verser une indemnité supplémentaire. Soit le licenciement n'est pas légalement possible et dans ce cas il ne devrait pas être mis en œuvre. Dans le projet de loi, une sorte d'hybridation a cependant été retenue. A partir de 2020, il existera un type de licenciement licite qui sera moins licite que d'autres et qui nécessitera donc une indemnisation supplémentaire.

\section{Evaluation}

Ce nouveau projet de loi a pour but de reprendre les choses là où les changements apportés en 2015 s'étaient arrêtés. Certaines idées, telles que l'égalisation des coûts entre les différents types de contrats, semblent utiles et justifiées. Ainsi, les employeurs ont le choix. La flexibilité reste possible, mais elle devient plus coûteuse, ce qui peut signifier qu'à l'avenir, elle sera utilisée en cas de nécessité plutôt que pour des raisons de commodité. Dans un pays où le travail flexible est devenu courant, cela peut représenter un gain énorme.

En revanche, certaines des autres propositions semblent offrir des perspectives beaucoup moins encourageantes. Nous sommes particulièrement sceptique sur l'augmentation de durée maximale de 24 à 36 mois des contrats à durée déterminée, renouvellement compris. Si les employeurs ne peuvent pas être incités à proposer des contrats à durée indéterminée après deux ans, même dans le contexte actuel du marché du travail, pourquoi devraient-ils être plus enclins à le faire dans un délai de trois ans? L'insertion du motif cumulatif de licenciement combiné à l'indemnité de licenciement supplémentaire semble une autre idée maladroite. Indépendamment de ce que dit le gouvernement, cela ne cadre pas avec le système actuel et, ce qui est encore plus important, cela crée un motif de mécontentement, puisque au fond «l'employeur peut faire ce qu'il veut tant qu'il est prêt à payer ». Avec de telles mesures, le droit du travail peut-il être toujours perçu comme un droit de protection?

10 TK 2018/19, 35 074, n³, p. 54.

11 Ibidem, p. 56 